\title{
A Systematic Review of Aspirin in Primary Prevention: Is It Time for a New Approach?
}

\author{
Carlos Brotons • Robert Benamouzig • \\ Krzysztof J. Filipiak • Volker Limmroth • \\ Claudio Borghi
}

Published online: 12 December 2014

(c) The Author(s) 2014. This article is published with open access at Springerlink.com

\begin{abstract}
Background and Objectives While evidence in support of aspirin use in secondary prevention is well documented, the role of aspirin in primary prevention remains unclear. We conducted a systematic literature review to evaluate aspirin use in cardiovascular disease (CVD) and cancer primary prevention, and consider whether aspirin's role is set to become more clearly defined based on past and prospective studies.

Data Sources Utilizing PubMed, the reviewers identified appropriate Medical Subject Headings (MeSH) terms to
\end{abstract}

All authors participated as paid consultants in a roundtable meeting on the use of aspirin in primary prevention, sponsored by Bayer HealthCare.

\section{Brotons $(\square)$}

Research Unit, Sardenya Primary Health Care Center, Biomedical Research Institute Sant Pau. Teaching Unit of Family Medicine ACEBA, Sardenya 466, 08025 Barcelona, Spain

e-mail: cbrotons@eapsardenya.cat

\section{R. Benamouzig}

Department of Gastroenterology, Avicenne Hospital, 125 rue de Stalingrad, 93000 Bobigny, France

\section{K. J. Filipiak}

First Department of Cardiology, Medical University of Warsaw, 1A Banacha St, 02-097 Warsaw, Poland

\section{Limmroth}

Department of Neurology, Cologne City Hospitals, University of Cologne, Ostmerheimerstrasse 200, 51109 Cologne, Germany

\section{Borghi}

Unità Operativa di Medicina Interna, Policlinico S. OrsolaMalpighi, University of Bologna, 40138 Bologna, Italy establish CVD-based studies, cancer-based studies, and studies on adherence.

Study Eligibility Criteria Date restrictions of May 31, 2008 to May 31, 2013 were applied to capture the most robust meta-analyses and randomized controlled trials. Websites of relevant EU and US scientific societies were used to identify the key guidelines for aspirin use in primary prevention of CVD, and ClinicalTrials.gov was used to establish future or ongoing trials.

Results Evidence in support of aspirin prophylaxis is conflicting, though some meta-analyses have underlined potential benefit in reducing cardiovascular events. Despite this apparent benefit, bleeding risk with aspirin is consistently higher versus control, and remains a concern. A reduction of cancer incidence and mortality after a least 3 and 5 years treatment, respectively, is also apparent with aspirin.

Conclusion Available data on aspirin in primary prevention suggest a modest benefit for patients at high risk of CVD, and a promising benefit for those at risk of cancer. Future studies should help to elucidate whether the benefit of aspirin outweighs risk in appropriate patient groups.

\section{Key Points}

Aspirin appears to provide a somewhat modest benefit in primary prevention of cardiovascular disease; more well-defined patient groups to establish those who would benefit most from regular aspirin are required.

Data from post hoc analyses of aspirin use in primary prevention of cancer are promising, especially in colorectal cancer; however, the extent of aspirin benefit in a high-burden disease requires further investigation in randomized controlled trials. 


\section{Introduction}

Both cardiovascular disease (CVD) and cancer are leading causes of mortality worldwide. In 2008, of the 57 million deaths that occurred globally, 36 million of these-almost two-thirds-were due to non-communicable diseases (NCDs), of which CVD and cancer were responsible for 17 million (47\% of all NCD deaths) and 7.6 million ( $21 \%$ of all NCD deaths), respectively [1].

Low-dose aspirin has been used for many years in the treatment and prevention of CVD. While the clinical benefits of aspirin for secondary prevention are well established, evidence on the role of aspirin for primary prevention is less clear. Current guidelines on the prophylactic use of aspirin vary widely, sometimes with conflicting recommendations. This may be due to varying interpretations of data from clinical trials of aspirin in primary prevention, which lead to differences in the perceived risk-benefit profile for aspirin.

Perception of the benefit versus risk ratio of aspirin for primary prevention may be influenced by the increasing body of evidence that aspirin reduces the risk of colorectal cancer (CRC) and other cancers [2, 3]. Recently revised guidelines on the management of Lynch syndrome (LS), which is the major, hereditary form of CRC, recommend the use of aspirin on the basis of long-term (i.e., 10 years) follow-up data from the CAPP2 trial, where aspirin significantly reduced the incidence of CRC in LS carriers [4].

As the overall benefit versus risk ratio is not yet clear, this paper systematically reviews the clinical evidence on the use of aspirin in primary prevention for CVD (with or without diabetes) and cancer. Moreover, it explores current use of aspirin in clinical practice and the clinical implications should subjects not adhere to their prophylactic regimen. Subsequent to these findings, we explore whether it is time for a new approach to the prophylactic use of aspirin by the most appropriate patient groups and make recommendations of our own while we await further outcome data from ongoing trials.

\section{Methods}

We conducted a systematic literature review, utilizing the PubMed database, to identify randomized controlled trials (RCTs) and meta-analyses of aspirin in the primary prevention of CVD (in subjects with and without diabetes) and cancer. We also investigated the impact of non-adherence and discontinuation of aspirin use once prescribed for primary prevention, and searched the websites of relevant EU and US scientific societies to identify the key guidelines for aspirin use in primary prevention of CVD.

\subsection{Literature Searches and Data Extraction}

The systematic review was conducted following the PRISMA guidelines. The search criteria can be observed in Table 1. The following search criteria to establish CVDbased studies on patients with or without diabetes were included: (clinical trial or meta-analysis), aspirin, primary prevention, and CVD. For the cancer search criteria, the following search terms were used: aspirin, cancer, and (meta-analysis or clinical trial). For all searches, the search was limited to human studies, English language was used, and date restrictions May 31, 2008 to May 31, 2013 were applied to allow for a 5-year assessment of the evidence base.

In order to be included, the meta-analyses had to (1) provide information on prophylactic aspirin use in relation to CVD or cancer incidence; and/or (2) have cancer in general or CRC incidence or mortality as an endpoint; (3) report original data and include hazard ratios (HRs), odds ratios (ORs), or relative risks (RRs) and their $95 \%$ confidence intervals (CIs); and (4) synthesize data from RCTs that compared aspirin therapy with placebo/vitamins in adults $>18$ years old with no history of CVD, or adults $>18$ years without cancer, though meta-analyses of observational and cohort studies were considered if they addressed point 1 of the criteria. Indeed, cancer is often studied observationally. Exclusion criteria for the metaanalyses included studies that were considered to be short term (less than 1 year of follow-up), and studies that were not published as full reports, such as conference abstracts and letters to editors. In addition, individual articles that were not of RCTs were excluded from this systematic review as cohort and case-control studies have limitations in the study design, which reduced the quality of the data obtained. Full text review was performed, and all authors determined the quality of articles. Risk of bias was considered and minimized by our chosen selection criteria.

For the search relating adherence to aspirin, the following search criteria were used: aspirin, primary prevention, CVD, and (prevalence or patient medication knowledge or patient adherence or medication adherence/persistence). Because of the low number of relevant publications retrieved, we analyzed all relevant studies that provided information on aspirin use in primary prevention, and therefore included cohort studies and cross-sectional studies. Additional publications identified in pertinent review articles were also reviewed. We understand the merit and limitations of these studies, and provide a narrative summation to help form our future hypotheses in the conclusions.

\subsection{Data Synthesis}

A narrative review of all extracted articles was undertaken; data reported from retrieved articles were either 
Table 1 Search criteria for systematic review

\begin{tabular}{|c|c|c|c|}
\hline Search strategy & $\begin{array}{l}\text { Number of articles } \\
\text { retrieved }\end{array}$ & Included & Excluded \\
\hline $\begin{array}{l}\text { Clinical trial[Publication Type] OR meta-analysis[Publication Type] AND “aspirin”[MeSH } \\
\text { Terms] AND “primary prevention”[MeSH Terms] AND “cardiovascular diseases”[MeSH } \\
\text { Terms] AND “humans”[MeSH Terms] AND English[Language] AND “2008/05/31”[PDAT]: } \\
\text { “2013/05/31"[PDAT] }\end{array}$ & 31 & 13 & 18 \\
\hline $\begin{array}{l}\text { ((“aspirin”[MeSH Terms] AND “neoplasms”[MeSH Terms]) AND ((“meta-analysis”[Publication } \\
\text { Type] OR “meta-analysis as topic”[MeSH Terms] OR “meta-analysis”[All Fields]) OR clinical } \\
\text { trial[Publication Type])) AND (“2008/05/31”[PDAT]: “2013/05/31”[PDAT]) AND ((“2008/05/ } \\
\text { 31”[PDAT]: “2013/05/31”[PDAT]) AND “humans”[MeSH Terms] AND English[lang]) }\end{array}$ & 80 & 6 & 74 \\
\hline $\begin{array}{l}\text { (((Aspirin[MeSH] AND primary prevention[MeSH] AND cardiovascular disease[MeSH] AND } \\
\text { (Prevalence[MeSH] OR Patient Medication Knowledge[MeSH] OR Patient Adherence[MESH] } \\
\text { OR Medication Adherence[MeSH] OR Medication Persistence[MeSH])))) AND (“2008/05/ } \\
\text { 31”[Date-Publication]: “2013/05/31”[Date-Publication]) }\end{array}$ & 13 & 3 & 10 \\
\hline
\end{tabular}

summarized or tabulated. The limitations for each study were considered and documented accordingly (e.g., publications bias and heterogeneity).

\section{Results}

\subsection{Aspirin Use in the Prevention of Cardiovascular Disease}

\subsubsection{Cardiovascular (CV) Events}

The search terms yielded 31 publications, of which nine were appropriate meta-analyses [5-13]. Ten primary studies are included in the meta-analyses, though only one of these was identified in our own search because of the publication date restrictions. The Early Treatment Diabetic Retinopathy Study (ETDRS) was included in three of the meta-analyses, though prevention of CVD was not the purpose of the study [14]. The nine trials that investigated aspirin for the primary prevention of cardiovascular (CV) events are listed in Table 2 [15-23]. Four additional subgroup or post hoc analyses for these trials were identified in our literature search, and are analyzed in the context of their original studies [24-27]. The meta-analyses and trials focused on patients with diabetes are described in a later section [9-13, 15, 19, 27].

The reasons for exclusion of the other 18 articles were varied; however, the most common reasons were review article ( $n=4$ articles), use of aspirin as a polypill $(n=2)$, use of aspirin in other indications $(n=3)$, and study of determinants of aspirin use $(n=2)$. The primary analysis of one retrieved meta-analysis [28] pooled data from primary and secondary prevention trials and was therefore excluded. No meta-analyses of observational studies of aspirin for primary CVD prevention were retrieved by the search terms. Inspection of one of the review articles obtained in the search [29] identified an additional relevant meta-analysis [30].

Details of the meta-analyses included are shown in Table $3[5-13,30]$. The meta-analyses report reductions in $\mathrm{CV}$ events with aspirin treatment compared with nonaspirin treatment; however, the differences are significant in only a small number of cases (Table 4) [5-13, 30]. For example, a $12 \%$ proportional reduction in serious vascular events [myocardial infarction (MI), stroke, or vascular death] was reported in Baigent et al. [5] (aspirin $0.51 \%$ vs. control $0.57 \%$ per year; $p=0.0001$ ), which was largely due to a $23 \%$ RR reduction for non-fatal MI (aspirin $0.18 \%$ vs. control $0.23 \%$ per year; $p<0.0001$ ) (Table 2). The meta-analysis by de Berardis et al. [10] also explored sex-specific differences; aspirin significantly reduced the risk of MI among men by $43 \%$ [three trials: Primary Prevention Project (PPP), ETDRS, and Physicians' Health Study (PHS); 3,126 participants; 265 events; RR 0.57 (95\% CI 0.34-0.94); $p=0.03$ ]. Conversely, no effect of aspirin was observed among women [three studies: Women's Health Study (WHS), PPP, ETDRS; 3,176 participants; 245 events; RR 1.08 (95\% CI 0.71-1.65); $p=0.71]$. In men, aspirin use was not associated with a reduced risk of stroke compared with placebo or no treatment [two trials: PPP and WHS; 2,593 participants; 93 events; RR 1.11 (95 \% CI 0.75-1.64); $p=0.61$ ], while in women, a risk reduction was observed [three studies: WHS, PPP, ETDRS; 3,176 participants; 127 events; RR 0.75 (95\% CI 0.37-1.53); $p=0.43$ ], albeit deemed not significant. Of note, Berger et al. [31] investigated the sexspecific differences in these outcomes in a previous metaanalysis; these findings influenced guideline development and are discussed later in the review.

There was no evidence that aspirin significantly reduces $\mathrm{CV}$ mortality in the five meta-analyses that did not focus on patients with diabetes (Table 4). A similar effect was seen for all-cause mortality, although in one meta-analysis, the 


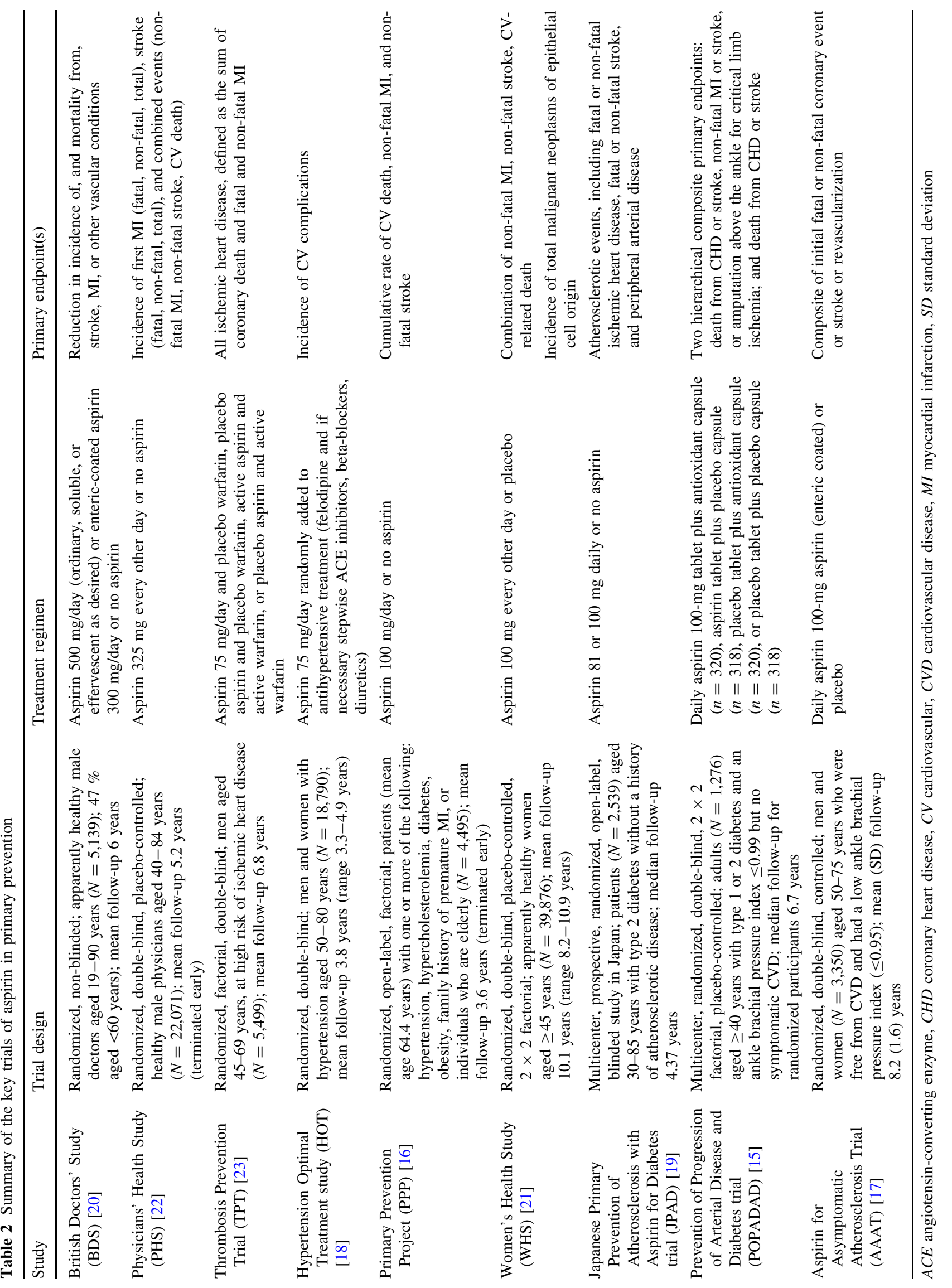




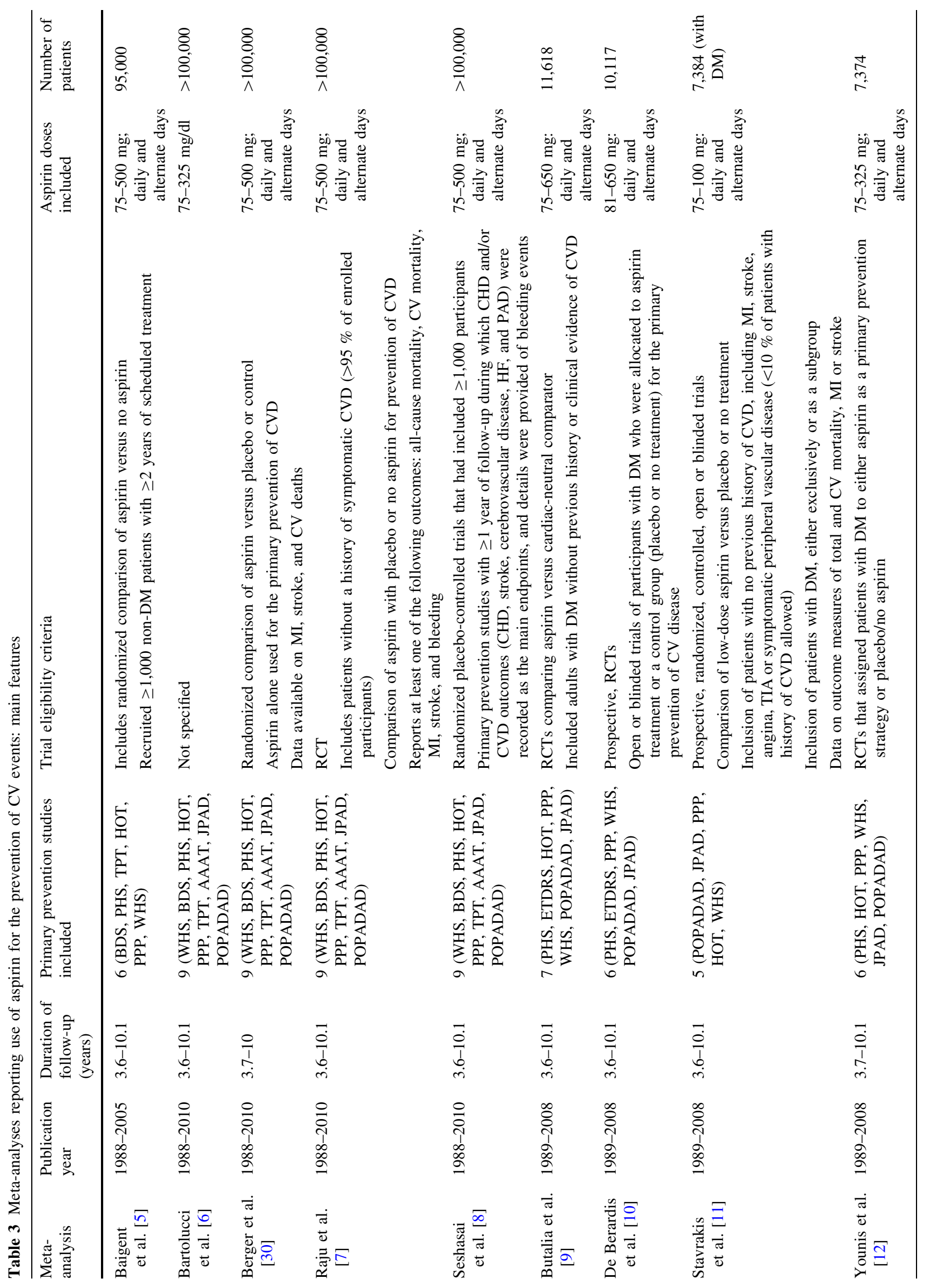




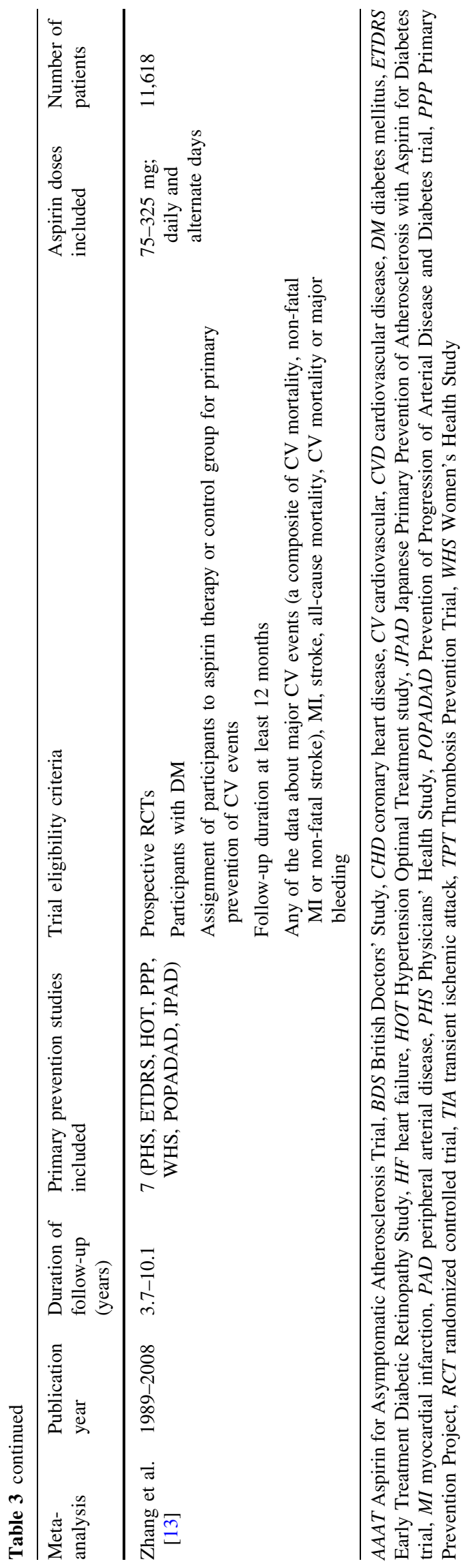

reduction in patients receiving aspirin was of borderline significance [RR 0.94 (95\% CI 0.88-1.00); $p=0.05$ ] [7].

In the WHS, the primary endpoint (major CV events) was not reduced significantly with aspirin use [RR 0.91 (95\% CI 0.80-1.03); $p=0.13$ ], though risk of all-cause stroke and ischemic stroke were significantly reduced [RR 0.83 (95\% CI 0.69-0.99), $p=0.04$, and RR $0.76(95 \%$ CI 0.63-0.93), $p=0.009$, respectively] [21]. A post hoc analysis of this study, utilizing existing risk scores and an 'optimal fit' model to predict treatment effects for individual women in terms of absolute risk reduction for major $\mathrm{CV}$ events with aspirin concluded that aspirin appeared to be ineffective in the majority of females, but that selective treatment of women $\geq 65$ years of age was of positive net benefit if the 10-year number-willing-to-treat (NWT) individuals was $>50$, but not if the NWT was $\leq 50$ [24]. Since almost all women $<65$ years of age $(99.2 \%)$ had $\leq 2 \%$ predicted absolute treatment effect, the authors of this study concluded that the place for aspirin in primary prevention in women is limited, with age being the strongest determinant of treatment effect. A separate subanalysis of the WHS aimed to explore the role of aspirin in CVD prevention in subjects with migraine; aspirin had similar protective effects on ischemic stroke for women with or without migraine [26]. By contrast, women with migraine with aura on aspirin had increased risk of MI. Authors acknowledge that this result should be cautiously interpreted.

In the primary RCT focusing on patients with hypertension [Hypertension Optimal Treatment study (HOT)], aspirin significantly reduced major $\mathrm{CV}$ events [RR 0.85 (95\% CI 0.73-0.99); $p=0.03$ ], particularly MI [RR 0.64 (95\% CI 0.49-0.85); $p=0.002]$, though did not affect the overall incidence of stroke [RR 0.98 (95 \% CI 0.78-1.24); $p=0.88$ ] [18]. In a post hoc subgroup analysis of this study, it was concluded that the benefit of aspirin is amplified in patients with hypertension and chronic kidney disease, with progressive (but non-linear) increases in aspirin benefit for major CV events, MI, all-cause stroke, CV mortality, and total mortality with decreasing renal function [25]. Aspirin was associated with pronounced, statistically significant reductions in the endpoints of $\mathrm{CV}$ mortality, all-cause stroke, and all-cause mortality in patients with an estimated glomerular filtration rate (GFR) of $\leq 45 \mathrm{ml} / \mathrm{min} / 1.73 \mathrm{~m}^{2}$. These findings indicate a greater benefit of aspirin in patients with reduced GFR, which is likely attributable to these patients being at higher CV risk.

\subsubsection{Bleeding Events}

Of the six meta-analyses focusing on data from non-diabetes patients (Table 4), bleeding events were analyzed in five. Baigent et al. [5] reported a significantly increased 


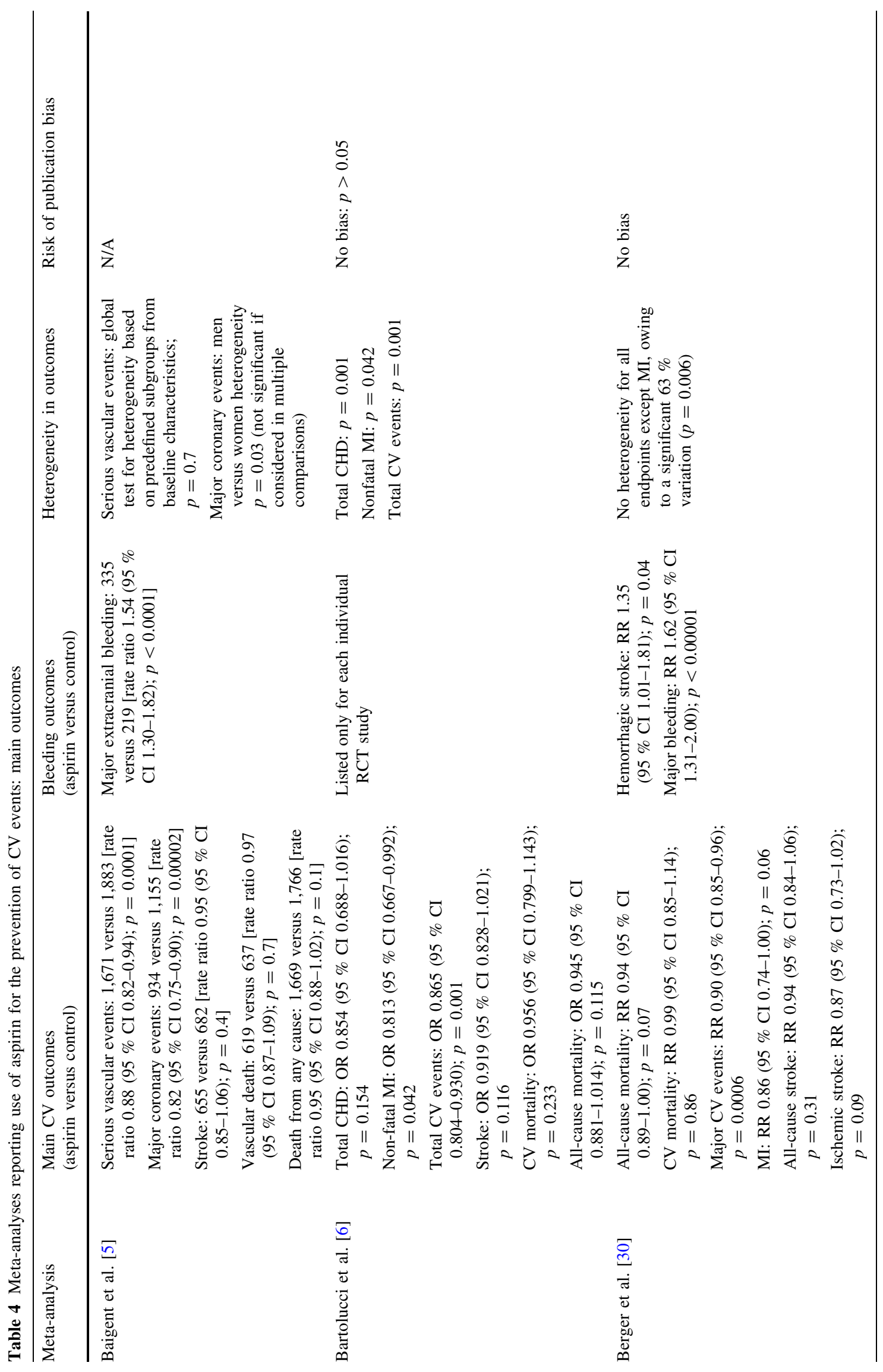




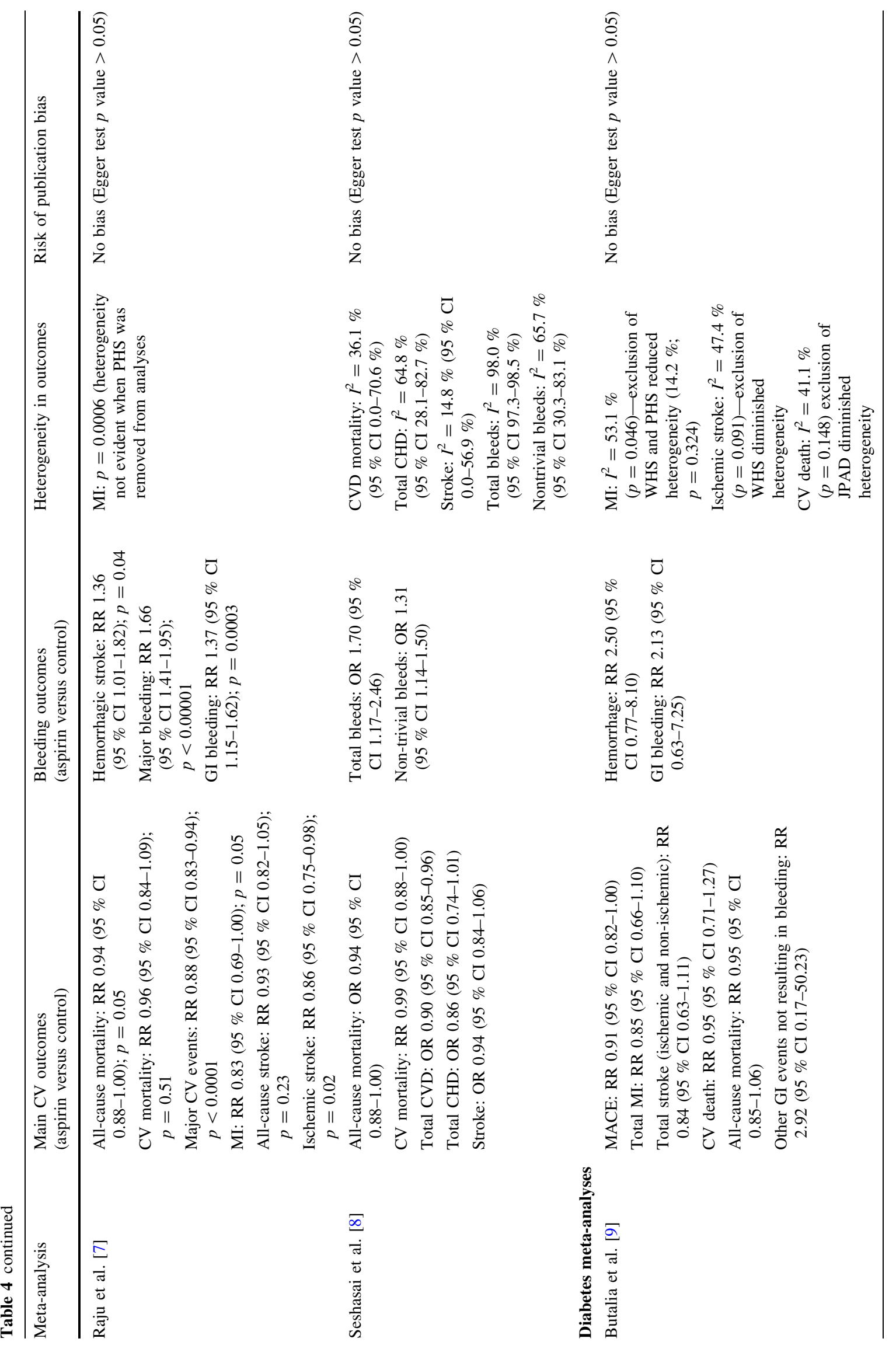




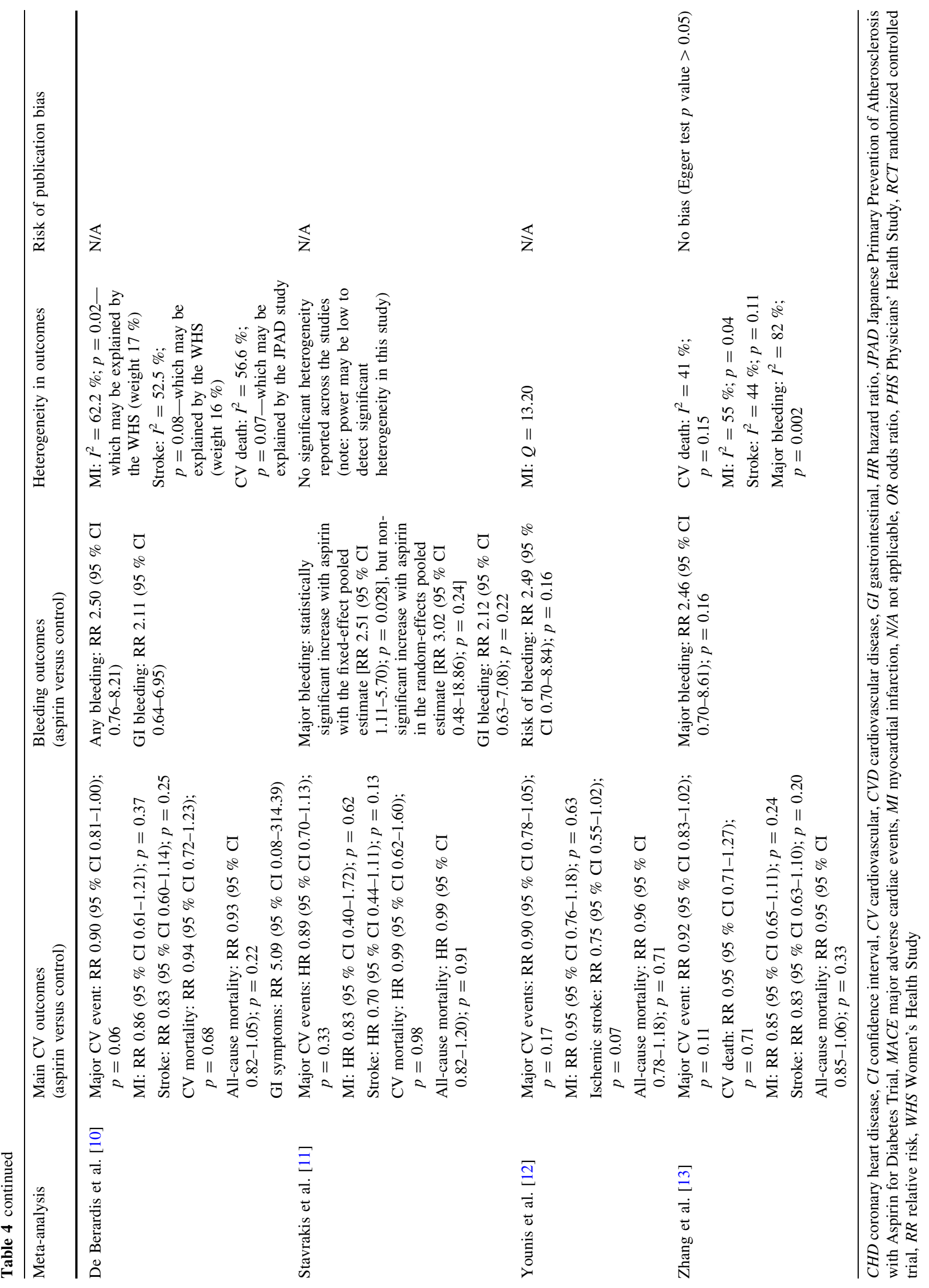


risk of major extracranial bleeding with aspirin [RR 1.54 (95\% CI 1.30-1.82); $p<0.0001]$, which was greater (non-significant) in patients with higher cholesterol. The excess risk of bleeds was mostly non-fatal. Perhaps by chance, fatal gastrointestinal (GI) or other fatal extracranial bleeds were lower in the aspirin group versus control [9 vs. 20; RR 0.48 (95 \% CI 0.17-1.34)] [5]. Of the other four meta-analyses, one reported significant increases in hemorrhagic stroke [RR 1.36 (95\% CI 1.01-1.82); $p=0.04$ ], major bleeding [RR $1.66 \quad(95 \%$ CI $1.41-1.95)$; $p<0.00001]$, and GI bleeding [RR $1.37 \quad(95 \%$ CI 1.15-1.62); $p=0.0003$ ] [7], while another reported comparable increases in hemorrhagic stroke and major bleeding [16]. The meta-analysis by Seshasai et al. [8] reported a $70 \%$ excess risk in total bleeds and $30 \%$ of non-trivial bleeds (Table 4). One did not report pooled data for bleeding events [6].

\subsection{Prophylactic Use of Aspirin for Prevention of CV Events in Patients With Diabetes}

\subsubsection{Events}

Table 4 shows the main $\mathrm{CV}$ outcomes from five metaanalyses that focused on data from patients with diabetes. None of these analyses reported a significant protective effect of aspirin for prevention of CV events [9-13]. All of the point estimates for the major $\mathrm{CV}$ and mortality-related outcomes in these meta-analyses favored aspirin, and in the meta-analysis of Butalia et al. [9], the reduction in major adverse cardiac events (MACE) was of borderline significance $(p=0.05)$ (Table 4$)$. No significant protective effect of aspirin has been observed in patients with diabetes, according to findings from the Japanese Primary Prevention of Atherosclerosis with Aspirin for Diabetes (JPAD) and Prevention of Progression of Arterial Disease and Diabetes (POPADAD) trials [15, 19]. In a subanalysis of the JPAD trial, in patients with diabetes, the outcomes of patients with hypertension were compared with those of normotensive patients [27]. While the incidence of cerebrovascular events was higher in patients with hypertension relative to those without [HR 2.84 (95\% CI 1.52-5.52); $p=0.0008]$, use of aspirin reduced the incidence rate in patients with hypertension to a similar level to that in patients without hypertension [HR $1.64 \quad(95 \%$ CI $0.83-3.29) ; p=0.15]$. However, aspirin use did not significantly decrease the incidence of cerebrovascular events in the group of patients with hypertension [27].

\subsubsection{Bleeding Events}

The meta-analyses that focused on patients with diabetes also reported incidence of bleeding events (Table 2).
Stavrakis et al. [11] reported a statistically significant increased risk of major bleeding with aspirin using a fixedeffect pooled estimate [RR 2.51 (95\% CI 1.11-5.70); $p=0.028]$; however, the difference was no longer significant using a random-effects pooled estimate [RR 3.02 (95\% CI 0.48-18.86); $p=0.24]$. Although the other metaanalyses reported numerically higher incidences of a range of bleeding categories with aspirin in patients with diabetes, none of these increases was statistically significant [9, 10, 12, 13]. This included the category of GI bleeding, for which a non-significant RR of 2.1 was reported in three of the meta-analyses [9-11]. Indeed, considering the bleeding risk in the two individual RCTs in patients with diabetes, in the POPADAD trial, the incidence of GI bleeding was not significantly increased in patients receiving aspirin [OR 0.90 (95\% CI 0.53-1.52); $p=0.69$ ] [15]. In the JPAD trial, there was no significant difference in the composite of hemorrhagic stroke and severe GI bleeding between patients in the aspirin and non-aspirin groups, based on 17 such events that occurred during the study [19].

\subsection{Prophylactic use of Aspirin in Colorectal Cancer}

The search terms retrieved 80 articles, of which six were relevant meta-analyses (Table 4) [32-37]. Two additional meta-analyses evaluated data on aspirin for the prevention of colorectal adenoma, but these publications were not analyzed further because they were secondary prevention trials $[38,39]$. Of the additional articles that were excluded, the most common reasons for exclusion were study on aspirin/non-steroidal anti-inflammatory drugs in other types of cancer ( $n=28$ articles), (pharmaco)genetic study $(n=5)$, and review article $(n=3)$. One article reported a systematic comparison of evidence from observational studies versus randomized trials [40]; however, there was no clear statement that the non-randomized studies included were primary prevention studies, and pooled data on the randomized trials in this article are already included in the meta-analysis by Rothwell et al. [34], which is included in Table 5. Five of the retrieved articles were RCTs [41-44].

\subsubsection{Incidence}

In a pooled analysis of six trials of daily low-dose aspirin for the primary prevention of $\mathrm{CV}$ events, during a followup period of 4-8 years, overall cancer incidence was reduced by $19 \%$ [OR 0.81 (95\% CI 0.67-0.98); $p=0.03$ ] after 3-4.9 years of aspirin use, increasing to $29 \%$ at $\geq 5$ years of use $[$ OR $0.71 \quad(95 \%$ CI $0.57-0.89)$; $p=0.003$, independent of age, sex, and smoking status [34]. In a related meta-analysis of five UK trials, a protective effect of aspirin against the development of new cancer was accompanied by a significant reduction in the 


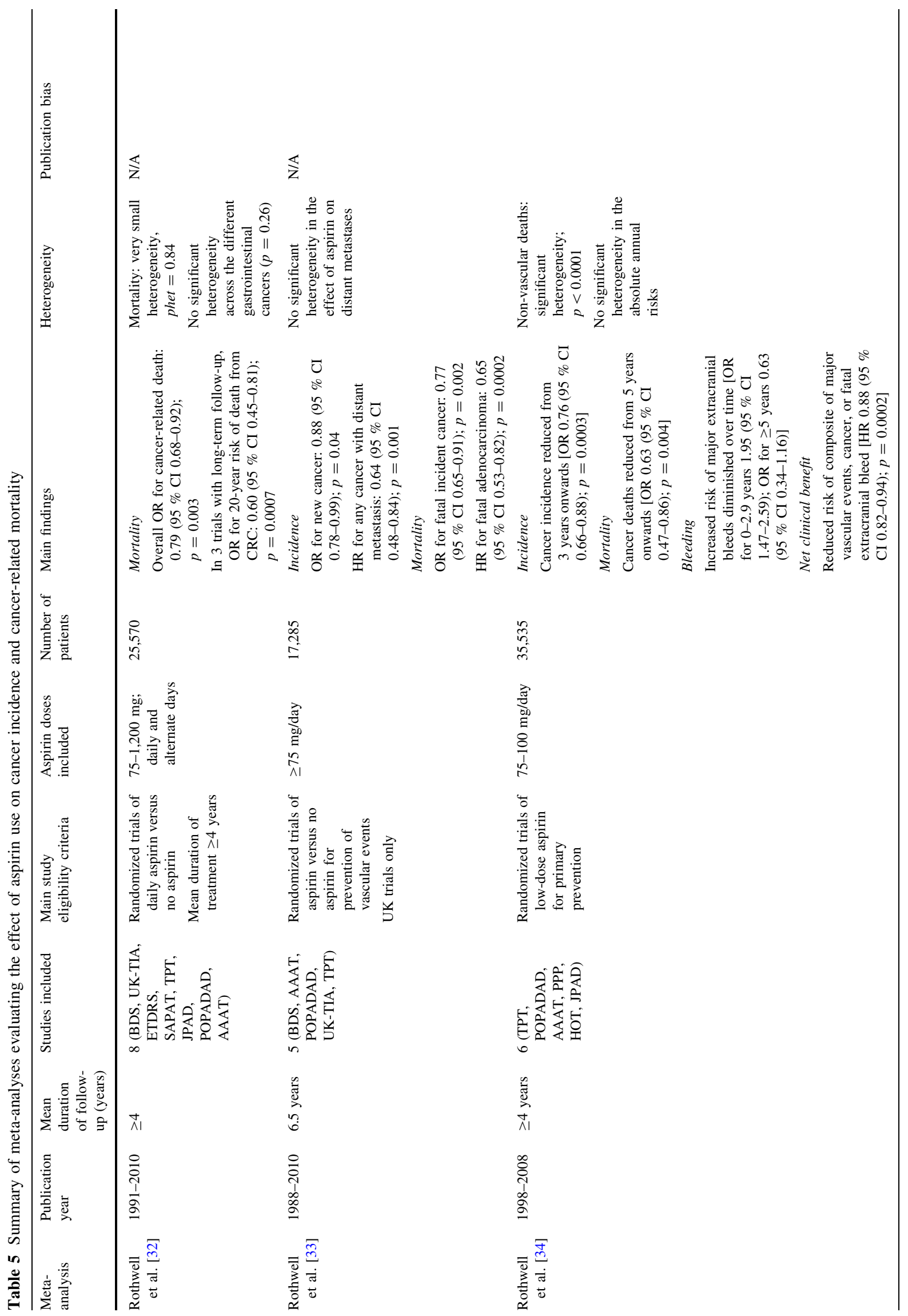


124

C. Brotons et al.

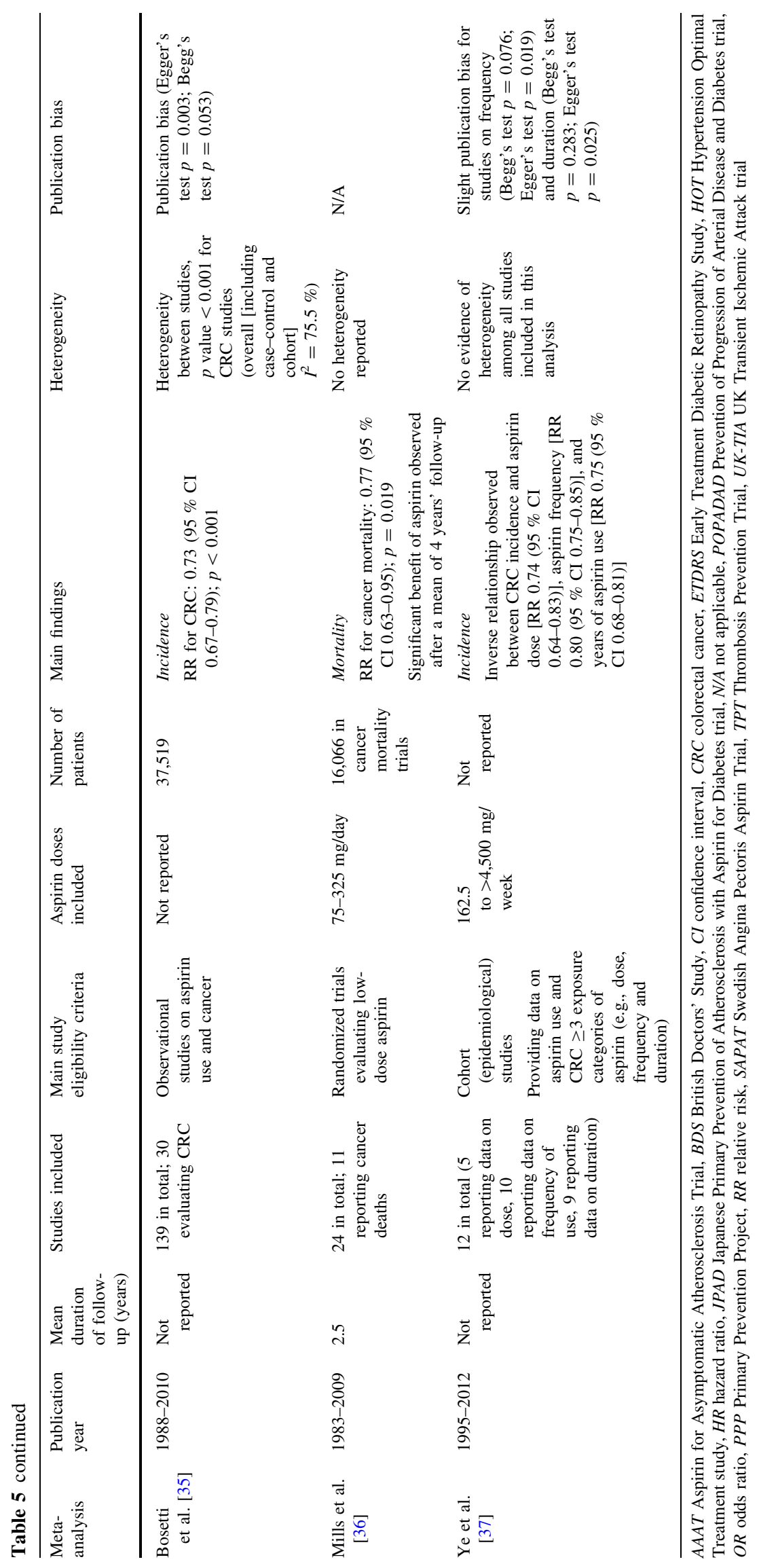

几 Adis 
incidence of cancer with distant metastasis [HR 0.64 (95\% CI $0.48-0.84) ; p=0.001$ ], with a significant benefit for adenocarcinomas, but not for other solid cancers [33]. Additional meta-analyses of observational and epidemiological studies provide supporting evidence for the protective effect of aspirin against cancer [35, 37]. In a metaanalysis of observational studies (case-control and cohort studies), there was a $27 \%$ reduction in the risk of CRC with regular aspirin use [RR $0.73 \quad(95 \%$ CI $0.73-0.79)$; $p<0.001]$; in terms of duration of aspirin use, the RR was $0.80(95 \%$ CI $0.71-0.91)$ for $<5$ years and 0.75 (95\% CI $0.70-0.80)$ for $\geq 5$ years $(P$ for heterogeneity $=0.369$ ) [35]. Overall, risk reductions were stronger in case-control studies versus cohort studies, corresponding with a significant heterogeneity observed $(p<0.001)$. The authors believed the high variability of aspirin use definitions across studies may partly explain the heterogeneity observed in risk estimates across studies. Moreover, this meta-analysis included case-control studies, which might be subject to selection and recall bias, leading to heterogeneous results. Publication bias was also significant. Despite the publication bias reported, a consistent cancer risk reduction was apparent, supporting the causality of this association.

In order to elucidate the public health implication of aspirin use in cancer prevention, the dose-risk and duration-risk relationships were assessed by Ye et al. [37] in a separate meta-analysis. Based on their findings, Ye et al. [37] concluded that the threshold effect is a dose of 75-325 mg daily and 2-7 times per week; there was a non- linear relation between dose of aspirin use and CRC risk ( $P$ for non-linearity $=0.020$ ). According to a random effects cubic spline model, there was stronger risk reduction for higher aspirin dose [RR 0.80 (95\% CI 0.74-0.88) for $325 \mathrm{mg}$ per day, and RR 0.74 (95\% CI 0.65-0.83) for $650 \mathrm{mg}$ per day]. This model, which included all studies on frequency of aspirin use (times per week), also indicated a non-linear relation between CRC risk and frequency of aspirin use ( $P$ for non-linearity $=0.007)$. Notably, there was a stronger risk reduction for people taking aspirin 7 times a week [RR 0.82 (95\% CI 0.78-0.87)] compared with twice per week [0.92 (95\% CI 0.88-0.95)]; this benefit was not strengthened in people taking aspirin more than 7 times a week [RR 0.82 (95\% CI 0.78-0.87) for 10 times per week] [37]. This meta-analysis included cohort studies only, with no evidence of heterogeneity observed across these studies.

\subsubsection{Mortality}

In a meta-analysis of eight trials including 25,570 patients, aspirin use significantly reduced the overall incidence of cancer-related death by $21 \%$ [32]. When the analysis was confined to the three trials with long-term follow-up ( $\geq 5$ years), a reduction in cancer mortality with aspirin was observed for all solid cancers [OR $0.80(95 \% \mathrm{CI}$ $0.72-0.88) ; p<0.0001]$ and GI cancers [OR 0.65 (95\% CI 0.54-0.78); $p<0.0001]$, but not hematological cancers [OR 1.03 (95\% CI 0.74-1.43); $p=0.87$ ] (Fig. 1) [20, 23,
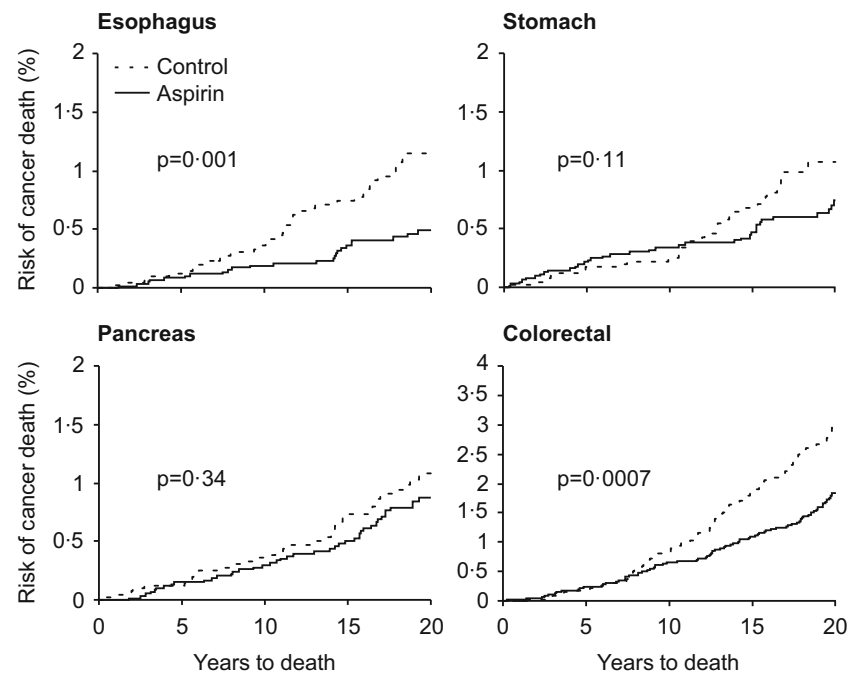
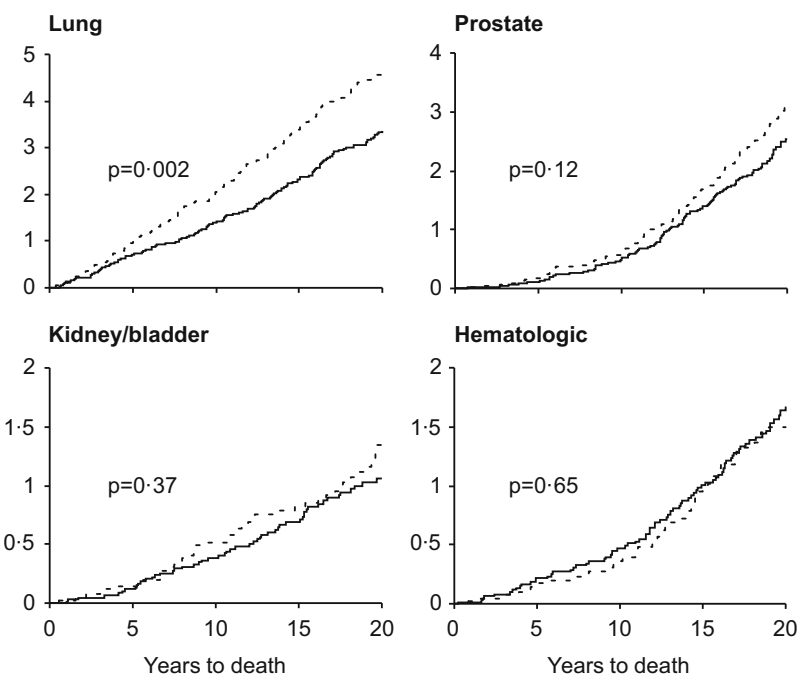

Number at risk

$\begin{array}{llllllllll}\text { Aspirin } & 6258 & 5816 & 5243 & 4485 & 2634 & 6258 & 5816 & 5243 & 4485\end{array}$ $\begin{array}{llllllllll}\text { Control } & 4244 & 3948 & 3545 & 3006 & 1493 & 4244 & 3948 & 3545 & 3006\end{array}$

Fig. 1 Effect of allocation to aspirin versus control on the 20-year risk of death due to the most common fatal cancers in 10,502 patients with scheduled treatment duration of $\geq 5$ years in three trials with long-term follow-up. Figure originally published in Rothwell et al. [32]. Reproduced with permission. Data are from a pooled analysis of $\begin{array}{lllllllllll}2634 & 6258 & 5816 & 5243 & 4485 & 2634 & 6258 & 5816 & 5243 & 4485 & 2634\end{array}$ $\begin{array}{lllllllllll}1493 & 4244 & 3948 & 3545 & 3006 & 1493 & 4244 & 3948 & 3545 & 3006 & 1493\end{array}$ three long-term follow-up trials [20, 23, 45]. All were randomized trials, during which patients received daily aspirin $(75-1,200 \mathrm{mg})$ for $\sim 4$ to 6.8 years (mean follow-up). Long-term data for deaths due to cancer following completion of the trials were collected via the national death certification and cancer registration systems 
$32,45]$. There was a significant effect of aspirin on the 20-year risk of death from CRC [OR 0.60 (95\% CI $0.45-0.81) ; p=0.0007]$, with a reduction in death evident from about 10 years onward. For deaths due to other cancers, such as esophageal or lung cancer, the benefit of aspirin was observed from 5 years onwards. In the metaanalysis of six trials of low-dose aspirin for primary prevention of $\mathrm{CV}$ events, cancer deaths were significantly reduced by $37 \%$ from 5 years onwards [OR $0.63(95 \%$ CI $0.47-0.86) ; p=0.004$ ] [34]. In a further meta-analysis, aspirin also significantly reduced overall cancer mortality [RR 0.77 (95\% CI 0.63-0.95); $p=0.019$ ] [36].

\subsubsection{Bleeding Events}

Of the included cancer-related meta-analyses, only one included results for adverse events attributable to aspirin use. In the meta-analysis of six trials, an increased risk of extracranial bleeding was observed with low-dose aspirin; however, the analysis of extracranial bleeding stratified by period of follow-up revealed that the risk of extracranial bleeding decreases over time, becoming comparable to that of placebo or no aspirin from 3 years onwards: $<3$ years, OR 1.95 (95\% CI $1.47-2.59) ; \quad 3-4.9$ years, 1.37 (0.87-2.14); $\geq 5$ years, $0.63(0.34-1.16) \quad(p=0.003$ for interaction) [34]. In this same analysis, fewer cases of fatal bleeding were associated with aspirin use, compared with controls [ $8 / 203$ vs. $15 / 132$, respectively; OR 0.32 (95\% CI $0.12-0.83) ; p=0.009$ ] [34].

\subsection{Aspirin Utilization}

Our search string for this topic identified 13 publications, of which only three publications were deemed relevant.

In a cross-sectional study of aspirin use in Wisconsin, USA, for primary prevention (with data from the Survey of the Health of Wisconsin), only $31 \%$ of the 268 participants (aged 35-74 years) for whom aspirin was indicated, according to United States Preventive Services Task Force (USPSTF) guidelines, were using it regularly (defined as $\geq 4$ times per week) [46]. Among these participants with an aspirin indication, older patients and women had a significantly higher likelihood of regular aspirin intake compared with younger participants and men (OR 1.07, $p<0.001$, and $3.49, p=0.021$, respectively) [46]. In a retrospective, cross-sectional cohort study in community outpatients in Italy, only $15.2 \%$ of 151,526 patients free from CVD received an aspirin prescription for primary prevention, despite being eligible for regular aspirin use according to the official guidelines stipulated by the Italian Medicine Agency. Indeed, patients with either type 2 diabetes and age $>40$ years or without type 2 diabetes but aged $\geq 55$ years (men) or $\geq 65$ years (women) with at least one risk factor (obesity, dyslipaemia, smoking cigarettes, hypertension, family history of CVD) were considered eligible [47]. A study conducted in the USA between 2005 and 2007 showed that the prevalence of regular aspirin ( $\geq 3$ days per week) use for primary prevention was only 31 and $44 \%$ for the 3,431 individuals at increased and high risk of coronary heart disease (CHD), respectively, with important racial and ethnic disparities [48].

\subsection{Aspirin Adherence}

Data on adherence to aspirin therapy for primary prevention of CV events are relatively scant. Indeed, the authors of a 2012 meta-analysis of studies that assessed patient adherence to $\mathrm{CV}$ preventive treatment for either primary or secondary prevention reported that no trials reporting adherence to aspirin therapy for primary prevention were identified [49].

A post hoc analysis of the WHS (study detailed in Table 2) [21] used statistical models to estimate the effect of continuous aspirin treatment on $\mathrm{CV}$ events [50]. Whereas the intent-to-treat population had small but nonsignificant reductions in total CV events [RR $0.91(95 \%$ CI $0.81-1.03) ; p=0.13$ ] and CV mortality [RR 0.95 (95\% CI 0.74-1.22); $p=0.68$ ], adjustment for aspirin non-compliance strengthened the effect of aspirin on CVD mortality, albeit without statistical significance [RR 0.76 (95 \% CI 0.54-1.08); $p=0.13]$.

\subsection{What Do the Guidelines Say?}

A summary of current guidelines for aspirin use in primary prevention is provided in Table 6 [51-55]. The majority of existing guidelines are based on meta-analyses and systematic reviews of the nine clinical trials for aspirin in primary prevention. The assessment and interpretation of these data can vary, leading to different outcomes for overall aspirin risk-benefit. Because of their basis on the nine aspirin primary prevention of CVD trials, most guidelines \{European Society of Cardiology (ESC) [52], USPSTF [55], American Heart Association/American Stroke Association (AHA/ASA) [54], American Diabetes Association (ADA) [51] \} only consider the benefits of a reduction in $\mathrm{CV}$ risk versus potential harms from bleeding. However, recommendations from different guideline bodies are sometimes conflicting. For example, the ESC 2012 guidelines state that aspirin cannot be recommended for primary prevention in patients without overt CVD, because of the increased risk of major bleeding (although it is recommended for some special patient groups, i.e., hypertensive patients without a history of CVD, patients with reduced renal function, and those at high $\mathrm{CV}$ risk) [52]. In contrast, the American College of Chest Physicians 
Table 6 Overview of current guidelines on the use of aspirin in primary prevention

\begin{tabular}{|c|c|}
\hline Organization & Recommendation \\
\hline European Society of Cardiology (ESC) [52] & $\begin{array}{l}\text { In patients without overt CVD, aspirin cannot be recommended in primary prevention } \\
\text { because of increased risk of major bleeding } \\
\text { Antiplatelet therapy may be considered in hypertensive patients without a history of CVD, } \\
\text { but with reduced renal function or at high CV risk } \\
\text { Antiplatelet therapy with aspirin is not recommended for people with diabetes who do not } \\
\text { have clinical evidence of atherosclerotic disease }\end{array}$ \\
\hline American Diabetes Association (ADA) [51] & $\begin{array}{l}\text { Consider aspirin therapy ( } 75-162 \mathrm{mg} / \text { day) as a primary prevention strategy in those with } \\
\text { type } 1 \text { or type } 2 \text { diabetes at increased CV risk }(10 \text {-year risk }>10 \%) \text {. This includes most } \\
\text { men aged }>50 \text { years or women aged }>60 \text { years who have at least one additional major risk } \\
\text { factor (family history of CVD), hypertension, smoking, dyslipidemia, or albuminuria }\end{array}$ \\
\hline $\begin{array}{l}\text { American College of Chest Physicians (ACCP) } \\
\text { [53] }\end{array}$ & Persons aged $\geq 50$ years without symptomatic CVD: low-dose aspirin $75-100 \mathrm{mg}$ daily \\
\hline $\begin{array}{l}\text { American Heart Association/American Stroke } \\
\text { Association (AHA/ASA) [54] }\end{array}$ & $\begin{array}{l}\text { Use of aspirin CV prophylaxis is recommended for persons whose risk is sufficiently high } \\
\text { for the benefits to outweigh the risks associated with treatment (i.e., 10-year risk of CV } \\
\text { event }=6-10 \% \text { ) } \\
\text { Aspirin can be useful for the prevention of a first stroke among women whose risk is } \\
\text { sufficiently high for the benefits to outweigh the risks associated with treatment } \\
\text { Aspirin is not useful for preventing a first stroke in persons at low risk } \\
\text { Aspirin is not useful for preventing a first stroke in persons with diabetes or diabetes plus } \\
\text { asymptomatic peripheral artery disease in the absence of any other CVD }\end{array}$ \\
\hline $\begin{array}{l}\text { United States Preventive Services Task Force } \\
\text { (USPSTF) [55] }\end{array}$ & $\begin{array}{l}\text { Encourage men aged } 45-79 \text { years to use aspirin when the potential benefit of a reduction in } \\
\text { MI outweighs the potential harm } \\
\text { Encourage women aged } 55-79 \text { years to use aspirin when the potential benefit of a reduction } \\
\text { of ischemic stroke outweighs the potential harm }\end{array}$ \\
\hline
\end{tabular}

$C V D$ cardiovascular disease, $C V$ cardiovascular, $M I$ myocardial infarction

(ACCP) 2012 guidelines suggest daily low-dose aspirin $(75-100 \mathrm{mg})$ in people aged $\geq 50$ years without symptomatic CVD [53]. The latter recommendations are based on an evaluation of the preventive benefit of aspirin on both CVD and cancer. The remaining guidelines cited in Table 6 recommend, in one way or another, the use of aspirin in specific groups of patients in primary prevention.

Both the USPSTF [55] and AHA/ASA [54] guidelines have specific recommendations for men and women. The USPTF guidelines recommend aspirin for men age 45-79 years when the potential benefit due to a reduction in MI outweighs the potential harm, while aspirin is only recommended for women age 55-79 years when the potential benefit of a reduction in ischemic strokes outweighs the potential harm. AHA guidelines also specifically recommend against aspirin use in men for stroke prevention, while stating that aspirin can be useful in women whose risk of stroke is sufficiently high enough for the benefits to outweigh the harms of treatment. When considering patients with diabetes, both ADA and AHA recommend aspirin therapy (75-162 mg/day) for primary prevention of heart disease for people with diabetes aged $>40$ years or who have additional risk factors for CVD and no contraindications to aspirin therapy [51, 54]. Currently, only the ACCP guidelines also mention data showing a reduction in cancer risk with aspirin [53].

\section{Discussion}

\subsection{Benefits}

Recent meta-analyses provide evidence of a modest benefit for aspirin in primary $\mathrm{CV}$ prevention, with a number of studies reporting statistically significant reductions in serious $\mathrm{CV}$ events, which did not extend to a reduction in $\mathrm{CV}$ or all-cause mortality across these studies [5-7]. The lack of statistical significance for outcomes related to mortality in some of these analyses might be attributed to the fact that the majority of patients included in these studies were not at high risk of CVD.

Although CV benefit was not observed in patients with diabetes enrolled in primary prevention trials, it has been suggested that these trials were not adequately powered to attain a reliable answer [56]. Indeed, in the JPAD trial, the investigators predicted 52 primary $\mathrm{CV}$ events per 1,000 person-years, but the actual rate was only 17 events per 1,000 person-years, therefore reducing the statistical relevance. Another possible explanation for the poor level of prevention afforded by aspirin in this patient population could be a reduced efficacy of low-dose aspirin in suppressing platelet function. It is hypothesized that faster resynthesis of platelets in patients with diabetes and thereby a faster resynthesis of megakaryocyte/platelet 
cyclooxygenase (COX) isozymes may allow sufficient recovery of COX-1 activity during the 24-h dosing interval (particularly between $12-24 \mathrm{~h}$ ), to overcome the antiplatelet effect of aspirin [57, 58]. Evidence from human pharmacokinetic studies suggests that twice-daily administration of low-dose aspirin in patients with diabetes reverses this pattern of insufficient COX-1 activity inhibition seen with a single daily aspirin dose [58]; however, trials with a clinical outcome parameter are required to confirm these preliminary findings. Another approach might be to increase the aspirin dose in resistant patients with diabetes. Results from a recent subanalysis of the Aspirin Versus/Or Clopidogrel in Aspirin-resistant Diabetics inflammation Outcomes (AVOCADO) trial showed that doubling the aspirin dose from $75 \mathrm{mg}$ to $150 \mathrm{mg}$ once daily improved platelet reactivity suppression in patients with type 2 diabetes and high platelet reactivity versus aspirin $75 \mathrm{mg}$ once daily [59].

\subsection{Cancer Benefit}

Evidence from primary RCTs demonstrate a reduction in the incidence of CRC and mortality from CRC with daily aspirin therapy [32-34, 36, 60]. In the meta-analysis by Rothwell et al. [32], aspirin reduced mortality by about $20 \%$. The reduction in cancer-related mortality observed was consistent across trials, despite the different populations included in the trials. Furthermore, the benefit of aspirin seems to be limited to certain cancers, with the most apparent benefit observed in adenocarcinomas, and by the length of treatment. Indeed, aspirin was only found to reduce cancer incidence from 3 years onwards [324 vs. 421 cases; OR 0.76 (95\% CI 0.66-0.88); $p=0.0003$ ], and benefit of aspirin on reducing cancer mortality was only apparent from 5 years onwards of treatment [66 vs. 104 deaths; OR 0.63 (95\% CI 0.47-0.86); $p=0.004$ ] [34]. Of note, as a reduced cancer mortality is not observed in people treated with warfarin, which has a similar bleeding profile to aspirin, it is unlikely that diagnostic procedures carried out early on in the investigations due to unexpected bleeding were the reason for lower cancer mortality [32].

Unlike the meta-analysis of RCTs observing no doseresponse effect on cancer outcomes [32], the meta-analysis of cohort studies by Ye et al. [37] reported an inverse relationship between CRC incidence and aspirin dose and aspirin frequency [37], with a 75- to 325-mg daily dose 2-7 times per week considered to provide optimal benefit. In the same analyses, there was also suggestion that longerterm ( $>5$ years) use of aspirin is necessary in order to demonstrate a protective effect on cancer risk [37], which is concordant with conclusions drawn by Rothwell et al. $[32,33]$ and consistent with 'true' prevention of the disease. On the topic of duration of aspirin exposure, a more clear cancer benefit in women has been observed with aspirin use lasting for 10 or more years, such as in the 82,911 women in the Nurses' Health Study [61]. The benefit observed in this study appears to be dose dependent, with a trend $(p<0.001$ for trend) in benefit observed by number of aspirin taken in a week [risk reduction in women taking aspirin $>14$ times a week $0.68 \quad(95 \%$ CI 0.49-0.95)]. Moreover, women who took more than 14 aspirin a week, 10 years preceding the study, had an even lower RR of 0.47 (95\% CI 0.31-0.71). That said, the incidence of major GI bleeding was also dose related, which again highlights the consideration required when determining the optimal dose of aspirin for benefit to supersede risk.

Similarly, and more recently, during the development of our review article, an update of the WHS on alternate-day aspirin use and cancer incidence, which was outside the time points given in our search criteria, was published [62]. Understanding of the apparent lag time before cancer benefit is observed; the WHS aimed to decipher whether alternate-day, low-dose aspirin use for 10 years [median active treatment of 10.3 years (range 8.2-10.9)] is associated with reduced risk of CRC in a post-treatment followup period (median follow-up of up to 18 years) [62]. A total of 33,682 women agreed to continue participation, with slightly more women in the aspirin arm compared with the placebo arm $[16,913(89.1 \%)$ vs. 16,769 $(88.2 \%) ; p=0.006]$. Unlike the original WHS [21] not showing clear CRC benefits during the active 10-year treatment phase, a post-treatment benefit in incidence of $\mathrm{CRC}$ was observed. Indeed, cancer incidence was lower in those taking aspirin [HR 0.80 (95\% CI $0.67-0.97)$; $p=0.021]$ than placebo, primarily due to a reduction in proximal colon cancer [HR 0.73 (95\% CI 0.55-0.95); $p=0.022]$, with the effect emerging after 10 years. The post-trial reduction in CRC was $42 \%$ [HR 0.58 (95\% $\mathrm{CI}=0.42-0.80) ; p<0.001] \quad$ [62]. Women who used aspirin during the active treatment phase but did not use aspirin post-trial had a $33 \%$ lower rate of CRC [HR 0.67 (95\% CI 0.43-1.02)], while those who continued with aspirin use had a $43 \%$ lower rate of CRC [HR 0.57 (95\% CI 0.35-0.93)].

Understanding that the effects of aspirin are not apparent until at least 3 years after starting aspirin treatment, a couple of studies have aimed to estimate the overall benefits and harms of aspirin over a 5- or 10-year period. A review of aspirin in cancer prevention conducted a 5-year risk analysis of the combined vascular and major bleeding events obtained from the ATT analysis of the six primary prevention trials (primary prevention trials of aspirin versus placebo) with a hypothetical $10 \%$ reduction in cancer incidence by age and sex. This analysis showed a net benefit of aspirin use for both men and women, with an 
even greater benefit for men aged $\geq 65$ years, compared with women or subjects of a younger age [3]. It is important to note, the authors' calculations of benefits after 5 years of use were based on an assumed reduction in overall cancer incidence with aspirin and not on empirical data. This may underestimate the reduction in cancer incidence that would occur with continued aspirin use beyond 10 years. A separate review that was published during the development of this article aimed to explore the 'best estimates' for individuals taking aspirin for 10 years by synthesizing data from available evidence on cancer, CVD (data obtained from ATT analysis) and its harm, and modeled these effects using general population data from the UK [63]. For individuals taking aspirin for 10 years, Cuzick et al. [63] estimated a 'relative' reduction of $\sim 9 \%$ in the number of men and $7 \%$ in the number women with a cancer, MI or stroke event over a 15-year period. Absolute reductions (conservative estimate) ranged from 0.68 to $3.09 \%$ in men and women; reductions in cancer incidence were estimated to account for $61-80 \%$ of the overall benefit, with reductions in CRC accounting for 30-36\% of it. Major bleeding events increased by between 0.16 and $0.81 \%$ from baseline rates of $0.57-2.37 \%$ over a 15 -year period. Based on these harms, the net relative benefit was calculated to be about $6 \%$. Relative reductions in cancer mortality were greater than that calculated for incidence (4\% relative reduction all deaths); concordant with trial data, no net reduction in CV-related deaths was reported. The relative benefit for mortality was more profound in men than in women, because of the lower baseline death rate from these diseases. Overall, these analyses suggest a net benefit for a minimum of 5 years of aspirin use, which heightens with 10 years of use in both men and women ranging between ages 50 and 65 .

So what renders aspirin seemingly cancer protective? The mechanism of action of aspirin in cancer prevention is not yet fully understood. Although the inflammatory-related gene COX-2 has been found to be over-expressed in adenomas, the protective effect of low-dose aspirin has been found to predominate in patients whose COX-2 expression was initially low [64]. Indeed, the main characteristics of the chemopreventive effect of low-dose aspirin are not consistent with either a direct inhibitory effect on COX-2 or with a COX-independent mechanism of action. Instead, it is hypothesized that aspirin may reduce the metastatic spread by acting through plateletmediated mechanisms; indeed, permanent inactivation of platelet COX-1 may play a key role in preventing colorectal adenoma formation, by suppressing the induction of COX-2 expression in adjacent cells [3, 65]. Aspirin may also have a role in directing modulation of oncogeneinduced expression of transcription factors and promotion of tumor cell apoptosis [66].

\subsection{Establishing the Benefit Versus Bleeding Risk}

The meta-analyses and randomized trial evidence demonstrate the increased risk of bleeding with aspirin is unequivocal, with most reporting a consistently high bleeding risk profile with aspirin use compared with control. However, data regarding the influence of age, underlying comorbidities (e.g., uncontrolled hypertension), and duration of use and dose of aspirin on bleeding risk are limited and conflicting.

Previous reviews of the literature have cited that increasing age per se should not be considered a significant risk factor for bleeding induced by low-dose aspirin [67]; rather, older patients in general have a much greater absolute risk of GI complications (GICs) than their younger counterparts [67], because there is a sharp increase in baseline risk of upper GICs (UGICs) in general among individuals aged $\geq 70$ years [68]. Moreover, evidence shows that rates of UGICs are higher in subjects with noncomplicated or complicated ulcers, compared with those without GI symptoms, and bleeding risk increases with age, irrespective of type of ulcer [68]. Notwithstanding, deaths from GI bleeding attributable to aspirin appear not to be increased [34]. Another aspect that should be considered is the possibility of concomitant Helicobacter pylori infection influencing bleeding risk. Evidence addressing the role of $H$. pylori and bleeding risk is currently conflicting $[69,70]$. Future studies (discussed in the next section) should help to qualify whether determining $H$. pylori status, in addition to age, sex and history of bleeds, is an important step towards profiling the right patient, with the least risk of bleeding, prior to commencement of aspirin prophylaxis.

\subsection{Limitations}

There exist some differences in trial design and patient characteristics among the RCTs that explored aspirin in CVD primary prevention; indeed, the primary endpoints and treatment regimens (e.g., aspirin once-daily vs. every other day) differed across each of the RCTs. Subsequent to these differences, when these RCTs were analyzed in the meta-analyses discussed in our review, statistical heterogeneity was reported among some of the efficacy and safety outcomes. Notably, when Butalia et al. [9] from their analyses of seven RCTs removed PHS and WHS, being the largest of the trials, heterogeneity substantially decreased. Thus, these meta-analyses have taught us that appropriately sized trials, with more specific patient segregation designed to consider different CVD risk profiles, are warranted to aid more definitive conclusions on the patient group that would likely benefit from aspirin in primary prevention of CVD.

Moreover, when considering the cancer benefit with aspirin use, although promising findings have been 
observed in meta-analyses of RCTs [32-34, 36, 60], a limitation of these analyses is that they are derived from studies analyzing aspirin in CVD protection where cancer was in most (with exception of WHS) not the primary outcome. In addition, we place caution on findings retrieved from a meta-analysis of observational studies; we need to consider the limitations inherent to observational studies, related in particular to measurement errors in the exposure to aspirin and the variability of aspirin use.

Regarding limitations of our own review, despite having rigorously reviewed the eligible articles extracted utilizing the specific search strings given in Table 1, we limited our search to the PubMed database, and restricted the search between two particular time points (between the years 2008 and 2013). However, we are confident of aspirin in primary prevention being well catalogued between these time points, and that the key articles of interest have been reviewed here. During the preparation of our manuscript, a robust systematic review by Sutcliffe et al. [71] re-evaluated aspirin use in primary prevention by estimating event rates, performing modeling on specific outcomes (i.e., all-cause mortality) and estimated heterogeneity in order to further rationalize the benefit of aspirin use according to the much scrutinized existing evidence base. Beyond analyzing the current data, our objective was to also review the evidence on studies on adherence of aspirin and on the recommendations of international guidelines, as well as presenting a hypothesis on the future direction of aspirin in primary prevention in anticipation of prospective and ongoing trials that may shed greater light on this subject.

\subsection{Future Direction and Study Needs}

A net benefit of aspirin that outweighs the risk of bleeding has been demonstrated when considering reductions in CV events and cancer together [34], thus one can speculate that aspirin may have a greater role in the primary prevention of both of these diseases in the near future. As such, it has been suggested that CRC benefits should be incorporated into CHD risk scores in order to determine the benefit-torisk ratio for aspirin use [72].

To identify those patients for whom aspirin is most appropriate, it is of our opinion a new individual approach to assessment that considers the combined benefits of aspirin as well as bleeding risk is required. Future criteria that might define a subject eligible for primary prevention with aspirin may include:

- Healthy subjects with a significantly increased risk of CVD using available risk charts.

- Healthy subjects with a significantly increased risk of cancer, in particular, CRC.
- Healthy subjects with a combined moderate risk of both CVD and cancer.

The ASPREE trial, an ongoing randomized, doubleblind study in the USA and Australia (expected to be completed by December 2018), should provide further evidence as to whether the benefits of daily aspirin use in healthy, elderly people outweigh the risks. The study is recruiting 19,000 healthy individuals aged over 70 years who will receive $100 \mathrm{mg}$ of aspirin or placebo daily for 5 years. The composite primary endpoint termed 'disability-free life' includes onset of dementia, total mortality, or persistent disability in at least one of the Katz Activities of Daily Living, and the secondary endpoints [all-cause specific mortality, fatal and non-fatal CV events, fatal and non-fatal cancer (excluding non-melanoma skin cancer), dementia, mild cognitive impairment, depression, physical disability, and clinically significant bleeding] will be compared. The results of this large trial should provide further insight into the risks versus overall benefits of aspirin prophylaxis. However, we note that any trial with short follow-up could potentially miss the net benefit, especially of fatal and non-fatal cancer. Indeed, aspirin for cancer prevention has a long lag time before benefits are observed, while conversely the harms appear in the early part of the treatment, particularly in those above 70 years of age.

To resolve some of the uncertainties around aspirin use in patients with diabetes, the results of ongoing clinical trials of aspirin in patients with diabetes, such as the Aspirin and Simvastatin Combination for CV Events Prevention Trial in Diabetes (ACCEPT-D; International Standard Randomized Controlled Trial Number: 48110081) and A Study of Cardiovascular Events iN Diabetes (ASCEND; ClinicalTrials.gov identifier: NCT00135226), are keenly awaited. Notably, these trials are more powered to assess the efficacy in reducing $\mathrm{CV}$ events than the past investigative trials discussed in our review, and should shed better light on the role aspirin plays in this clinical scenario.

Strategies to improve the bleeding profile of aspirin are recommended for appropriate patients. For example, coadministration of aspirin with a gastroprotective drug, such as proton pump inhibitors, should be considered for patients at high risk of UGICs, as these can lead to nonadherence and discontinuation with aspirin therapy [73]. While there are conflicting data on the role of $H$. pylori as a risk factor for UGICs with low-dose aspirin [69, 70], the ongoing large-scale outcomes study Helicobacter Eradication Aspirin Trial (HEAT; ClinicalTrials.gov identifier NCT01506986) promises to provide more definitive information on the relationship between $H$. pylori infection and GI bleeding during aspirin use. However, the limitation 
of this trial is that it investigates current users of aspirin and does not account for the proportion of individuals who discontinue aspirin because of GI symptoms.

Aspirin is currently underutilized in primary prevention [47]. The full extent, reasons, and consequences of aspirin underuse is poorly understood; reasons for underutilization may be related to the patient, condition, therapy or healthcare system. The highest-risk patients are frequently older with cognitive or physical impairments, or multiple comorbidities requiring polypharmacy. They may also hold negative perceptions about aspirin bleeding risk or have a low motivation to take indefinite therapy.

Since 'healthy' individuals are asymptomatic from a CV viewpoint, and the benefits of aspirin for primary prevention are not visible, while adverse events are obvious, counseling is important and should include discussion of the benefits of prophylaxis with patients prior to starting aspirin use. Adherence should be monitored and effective strategies to encourage therapeutic adherence employed. There is also a need for greater public education on aspirin for primary prevention, including information on the risks of inappropriate aspirin use and the consequences of discontinuation [47].

\section{Conclusions}

Emerging data from prospective studies of the protective effect of aspirin on cancer, combined with its proven effect on reduction of $\mathrm{CV}$ events, are likely to be included in future evaluations of benefit versus risk conducted by guideline bodies. This may influence recommendations on the use of aspirin for primary prevention, not only for CV events, but also cancer. Since CVD and cancer share a number of common risk factors, including a sedentary lifestyle, obesity, diabetes, poor diet, and smoking [74], and also share some disease mechanisms, a combined approach to prevention would be logical. However, unlike CVD risk assessment, there is some difficulty in assessing cancer risk (except for cases with familial and genetic factors) in clinical practice. Thus, profiling a patient who would benefit from aspirin in primary prevention of cancer should be based on their risk of bleeding rather the risk factors for cancer.

Acknowledgments The authors would like to thank Professors Carlo Patrono, Héctor Bueno, and Peter Elwood for their invaluable contribution to discussions at the roundtable meeting held in Paris, France, December 2012. This meeting was funded by Bayer HealthCare. Editorial assistance was provided by Victoria Panagakis of Fishawack Communications Ltd and funded by Bayer HealthCare.

Conflicts of interest Dr Carlos Brotons is a member of the Executive Committee for the ARRIVE trial, which is sponsored by Bayer HealthCare. Professor Limmroth has received honoraria as a speaker or consultant from Allergan, Bayer HealthCare, BiogenIdec, BristolMyers Squibb, Novartis, Pfizer, Roche, Merck Serono, and Teva. All authors participated as paid consultants in a roundtable meeting on the use of aspirin in primary prevention, sponsored by Bayer HealthCare. The authors declare that there are no other conflicts of interest, that they have full control of the primary data, and that they agree to allow the journal to review the data if requested.

Role of the funding source The contents of the review article entirely reflect the opinion of the authors. Carlos Brotons selected the search criteria and determined the methodology. All authors reviewed the literature and were each involved in the preparation of the manuscript. All authors critically reviewed the manuscript and approved the final content. For the initial meeting of the authors (a roundtable discussion), Bayer provided an unrestricted educational grant.

Open Access This article is distributed under the terms of the Creative Commons Attribution Noncommercial License which permits any noncommercial use, distribution, and reproduction in any medium, provided the original author(s) and the source are credited.

\section{References}

1. World Health Organization. NCD mortality and morbidity. http:// www.who.int/gho/ncd/mortality_morbidity/en. Accessed 12 Mar 2014.

2. Rothwell PM. Aspirin in prevention of sporadic colorectal cancer: current clinical evidence and overall balance of risks and benefits. Recent Results Cancer Res. 2013;191:121-42.

3. Thun MJ, Jacobs EJ, Patrono C. The role of aspirin in cancer prevention. Nat Rev Clin Oncol. 2012;9:259-67.

4. Vasen HF, Blanco I, Aktan-Collan K, et al. Revised guidelines for the clinical management of Lynch syndrome (HNPCC): recommendations by a group of European experts. Gut. 2013;62:812-23.

5. Baigent C, Blackwell L, Collins R, et al. Aspirin in the primary and secondary prevention of vascular disease: collaborative metaanalysis of individual participant data from randomised trials. Lancet. 2009;373:1849-60.

6. Bartolucci AA, Tendera M, Howard G. Meta-analysis of multiple primary prevention trials of cardiovascular events using aspirin. Am J Cardiol. 2011;107:1796-801.

7. Raju N, Sobieraj-Teague M, Hirsh J, et al. Effect of aspirin on mortality in the primary prevention of cardiovascular disease. Am J Med. 2011;124:621-9.

8. Seshasai SR, Wijesuriya S, Sivakumaran R, et al. Effect of aspirin on vascular and nonvascular outcomes: meta-analysis of randomized controlled trials. Arch Intern Med. 2012;172:209-16.

9. Butalia S, Leung A, Ghali W, et al. Aspirin effect on the incidence of major adverse cardiovascular events in patients with diabetes mellitus: a systematic review and meta-analysis. Cardiovasc Diabetol. 2011;10:25.

10. De Berardis G, Sacco M, Strippoli GF, et al. Aspirin for primary prevention of cardiovascular events in people with diabetes: metaanalysis of randomised controlled trials. BMJ. 2009;339:b4531.

11. Stavrakis S, Stoner JA, Azar M, et al. Low-dose aspirin for primary prevention of cardiovascular events in patients with diabetes: a meta-analysis. Am J Med Sci. 2011;341:1-9.

12. Younis N, Williams S, Ammori B, et al. Role of aspirin in the primary prevention of cardiovascular disease in diabetes mellitus: a meta-analysis. Expert Opin Pharmacother. 2010;11:1459-66.

13. Zhang C, Sun A, Zhang P, et al. Aspirin for primary prevention of cardiovascular events in patients with diabetes: a meta-analysis. Diabetes Res Clin Pract. 2010;87:211-8. 
14. Kassoff A, Buzney SM, McMeel J, et al. Aspirin effects on mortality and morbidity in patients with diabetes mellitus: Early Treatment Diabetic Retinopathy Study report 14. JAMA. 1992;268:1292-300.

15. Belch J, MacCuish A, Campbell I, et al. The Prevention Of Progression of Arterial Disease And Diabetes (POPADAD) trial: factorial randomised placebo controlled trial of aspirin and antioxidants in patients with diabetes and asymptomatic peripheral arterial disease. BMJ. 2008;337:a1840.

16. de Gaetano G. Low-dose aspirin and vitamin $\mathrm{E}$ in people at cardiovascular risk: a randomised trial in general practice. Collaborative Group of the Primary Prevention Project. Lancet. 2001;357:89-95.

17. Fowkes FG, Price JF, Stewart MC, et al. Aspirin for prevention of cardiovascular events in a general population screened for a low ankle brachial index: a randomized controlled trial. JAMA. 2010;303:841-8.

18. Hansson L, Zanchetti A, Carruthers SG, et al. Effects of intensive blood-pressure lowering and low-dose aspirin in patients with hypertension: principal results of the Hypertension Optimal Treatment (HOT) randomised trial. HOT Study Group. Lancet. 1998;351:1755-62.

19. Ogawa H, Nakayama M, Morimoto T, et al. Low-dose aspirin for primary prevention of atherosclerotic events in patients with type 2 diabetes: a randomized controlled trial. JAMA. 2008;300: 2134-41.

20. Peto R, Gray R, Collins R, et al. Randomised trial of prophylactic daily aspirin in British male doctors. Br Med J (Clin Res Ed). 1988;296:313-6.

21. Ridker PM, Cook NR, Lee IM, et al. A randomized trial of lowdose aspirin in the primary prevention of cardiovascular disease in women. N Engl J Med. 2005;352:1293-304.

22. Steering Committee of the Physicians' Health Study Research Group. Final report on the aspirin component of the ongoing Physicians' Health Study. Steering Committee of the Physicians' Health Study Research Group. N Engl J Med. 1989;321:129-35.

23. The Medical Research Council's General Practice Research Framework. Thrombosis Prevention Trial: randomised trial of low-intensity oral anticoagulation with warfarin and low-dose aspirin in the primary prevention of ischaemic heart disease in men at increased risk. The Medical Research Council's General Practice Research Framework. Lancet. 1998;351:233-41.

24. Dorresteijn JAN, Visseren FLJ, Ridker PM, et al. Aspirin for primary prevention of vascular events in women: individualized prediction of treatment effects. Eur Heart J. 2011;32:2962-9.

25. Jardine MJ, Ninomiya T, Perkovic V, et al. Aspirin is beneficial in hypertensive patients with chronic kidney disease: a post-hoc subgroup analysis of a randomized controlled trial. J Am Coll Cardiol. 2010;56:956-65.

26. Kurth T, Diener HC, Buring JE. Migraine and cardiovascular disease in women and the role of aspirin: subgroup analyses in the Women's Health Study. Cephalalgia. 2011;31:1106-15.

27. Soejima H, Ogawa H, Morimoto T, et al. Aspirin reduces cerebrovascular events in type 2 diabetic patients with poorly controlled blood pressure. Subanalysis from the JPAD trial. Circ J. 2012;76:1526-32.

28. Simpson SH, Gamble JM, Mereu L, et al. Effects of aspirin dose on mortality and cardiovascular events in people with diabetes: a meta-analysis. J Gen Intern Med. 2011;26:1336-44.

29. Tagliabue L, Dipaola F, Perego F, et al. Aspirin for the primary prevention of cardiovascular diseases. Intern Emerg Med. 2012;7:375-9.

30. Berger JS, Lala A, Krantz MJ, et al. Aspirin for the prevention of cardiovascular events in patients without clinical cardiovascular disease: a meta-analysis of randomized trials. Am Heart J. 2011;162(115-24):e2.
31. Berger JS, Roncaglioni MC, Avanzini F, et al. Aspirin for the primary prevention of cardiovascular events in women and men: a sex-specific meta-analysis of randomized controlled trials. JAMA. 2006;295:306-13 (Erratum in: JAMA. 2006;295:2002).

32. Rothwell PM, Fowkes FG, Belch JF, et al. Effect of daily aspirin on long-term risk of death due to cancer: analysis of individual patient data from randomised trials. Lancet. 2011;377:31-41.

33. Rothwell PM, Wilson M, Price JF, et al. Effect of daily aspirin on risk of cancer metastasis: a study of incident cancers during randomised controlled trials. Lancet. 2012;379:1591-601.

34. Rothwell PM, Price JF, Fowkes FG, et al. Short-term effects of daily aspirin on cancer incidence, mortality, and non-vascular death: analysis of the time course of risks and benefits in 51 randomised controlled trials. Lancet. 2012;379:1602-12.

35. Bosetti C, Rosato V, Gallus S, et al. Aspirin and cancer risk: a quantitative review to 2011. Ann Oncol. 2012;23:1403-15.

36. Mills EJ, Wu P, Alberton M, et al. Low-dose aspirin and cancer mortality: a meta-analysis of randomized trials. Am J Med. 2012;125:560-7.

37. Ye X, Fu J, Yang Y, et al. Dose-risk and duration-risk relationships between aspirin and colorectal cancer: a meta-analysis of published cohort studies. PLoS One. 2013;8:e57578.

38. Cole BF, Logan RF, Halabi S, et al. Aspirin for the chemoprevention of colorectal adenomas: meta-analysis of the randomized trials. J Natl Cancer Inst. 2009;101:256-66.

39. Gao F, Liao C, Liu L, et al. The effect of aspirin in the recurrence of colorectal adenomas: a meta-analysis of randomized controlled trials. Colorectal Dis. 2009;11:893-901.

40. Algra AM, Rothwell PM. Effects of regular aspirin on long-term cancer incidence and metastasis: a systematic comparison of evidence from observational studies versus randomised trials. Lancet Oncol. 2012;13:518-27.

41. Burn J, Bishop DT, Chapman PD, et al. A randomized placebocontrolled prevention trial of aspirin and/or resistant starch in young people with familial adenomatous polyposis. Cancer Prev Res (Phila). 2011;4:655-65.

42. Burn J, Gerdes AM, Macrae F, et al. Long-term effect of aspirin on cancer risk in carriers of hereditary colorectal cancer: an analysis from the CAPP2 randomised controlled trial. Lancet. 2011;378:2081-7.

43. Barry EL, Sansbury LB, Grau MV, et al. Cyclooxygenase-2 polymorphisms, aspirin treatment, and risk for colorectal adenoma recurrence-data from a randomized clinical trial. Cancer Epidemiol Biomarkers Prev. 2009;18:2726-33.

44. Burn J, Bishop DT, Mecklin JP, et al. Effect of aspirin or resistant starch on colorectal neoplasia in the Lynch syndrome. N Engl J Med. 2008;359:2567-78.

45. Farrell B, Godwin J, Richards S, et al. The United Kingdom Transient Ischaemic Attack (UK-TIA) aspirin trial: final results. J Neurol Neurosurg Psychiatry. 1991;54:1044-54.

46. Vanwormer JJ, Greenlee RT, McBride PE, et al. Aspirin for primary prevention of CVD: are the right people using it? J Fam Pract. 2012;61:525-33.

47. Filippi A, Bianchi C, Parazzini F, et al. A national survey on aspirin patterns of use and persistence in community outpatients in Italy. Eur J Cardiovasc Prev Rehabil. 2011;18:695-703.

48. Sanchez DR, Diez Roux AV, Michos ED, et al. Comparison of the racial/ethnic prevalence of regular aspirin use for the primary prevention of coronary heart disease from the multi-ethnic study of atherosclerosis. Am J Cardiol. 2011;107:41-6.

49. Naderi SH, Bestwick JP, Wald DS. Adherence to drugs that prevent cardiovascular disease: meta-analysis on 376,162 patients. Am J Med. 2012;125(882-87):e1.

50. Cook NR, Cole SR, Buring JE. Aspirin in the primary prevention of cardiovascular disease in the Women's Health Study: effect of noncompliance. Eur J Epidemiol. 2012;27:431-8. 
51. American Diabetes Association. Standards of medical care in diabetes-2013. Diabetes Care. 2013;36(Suppl 1):S11-66.

52. Perk J, De Backer G, Gohlke H, European Guidelines on cardiovascular disease prevention in clinical practice (version, 2012), et al. The Fifth Joint Task Force of the European Society of Cardiology and Other Societies on Cardiovascular Disease Prevention in Clinical Practice (constituted by representatives of nine societies and by invited experts). Eur Heart J. 2012; 2012(33):1635-701.

53. Vandvik PO, Lincoff AM, Gore JM, et al. Primary and secondary prevention of cardiovascular disease: antithrombotic therapy and prevention of thrombosis, 9th ed: American College of Chest Physicians evidence-based clinical practice guidelines. Chest. 2012;141:e637S-68S.

54. Goldstein LB, Bushnell CD, Adams RJ, et al. Guidelines for the primary prevention of stroke: a guideline for healthcare professionals from the American Heart Association/American Stroke Association. Stroke. 2011;42:517-84.

55. US Preventive Services Task Force. Aspirin for the prevention of cardiovascular disease: U.S. Preventive Services Task Force recommendation statement. Ann Intern Med. 2009;150:396-404.

56. Farkouh ME, Fuster V. Diabetes and aspirin: beware of underpowered negative trials. Nat Clin Pract Cardiovasc Med. 2009;6:1.

57. Patrono C. Diabetes: does aspirin increase the risk of major bleeds? Nat Rev Cardiol. 2012;9:495-6.

58. Rocca B, Santilli F, Pitocco D, et al. The recovery of platelet cyclooxygenase activity explains interindividual variability in responsiveness to low-dose aspirin in patients with and without diabetes. J Thromb Haemost. 2012;10:1220-30.

59. Rosiak M, Postula M, Kaplon-Cieslicka A, et al. The effect of doubling the dose of acetylsalicylic acid (ASA) on platelet function parameters in patients with type 2 diabetes and platelet hyperreactivity during treatment with $75 \mathrm{mg}$ of ASA: a subanalysis of the AVOCADO study. Kardiol Pol. 2013;71:552-7.

60. Rothwell PM, Wilson M, Elwin CE, et al. Long-term effect of aspirin on colorectal cancer incidence and mortality: 20-year follow-up of five randomised trials. Lancet. 2010;376:1741-50.

61. Chan AT, Giovannucci EL, Meyerhardt JA, et al. Long-term use of aspirin and nonsteroidal anti-inflammatory drugs and risk of colorectal cancer. JAMA. 2005;294:914-23.

62. Cook NR, Lee IM, Zhang SM, et al. Alternate-day low-dose aspirin and cancer risk: long-term observational follow-up of a randomized trial. Ann Intern Med. 2013;159:77-85.
63. Cuzick J, Thorat MA, Bosetti C, et al. Estimates of benefits and harms of prophylactic use of aspirin in the general population. Ann Oncol. 2014 (Epub ahead of print).

64. Benamouzig R, Uzzan B, Martin A, et al. Cyclooxygenase-2 expression and recurrence of colorectal adenomas: effect of aspirin chemoprevention. Gut. 2010;59:622-9.

65. Patrono C, Patrignani P, Garcia Rodriguez LA. Cyclooxygenaseselective inhibition of prostanoid formation: transducing biochemical selectivity into clinical read-outs. J Clin Invest. 2001;108:7-13.

66. Chan AT, Arber N, Burn J, et al. Aspirin in the chemoprevention of colorectal neoplasia: an overview. Cancer Prev Res (Phila). 2012;5:164-78.

67. Laine L. Review article: gastrointestinal bleeding with low-dose aspirin-what's the risk? Aliment Pharmacol Ther. 2006;24: 897-908.

68. Patrono C, Garcia Rodriguez LA, Landolfi R, et al. Low-dose aspirin for the prevention of atherothrombosis. N Engl $\mathrm{J}$ Med. 2005;353:2373-83.

69. Fletcher EH, Johnston DE, Fisher CR, et al. Systematic review: Helicobacter pylori and the risk of upper gastrointestinal bleeding risk in patients taking aspirin. Aliment Pharmacol Ther. 2010;32:831-9.

70. Chan FK, Ching JY, Suen BY, et al. Effects of Helicobacter pylori infection on long-term risk of peptic ulcer bleeding in lowdose aspirin users. Gastroenterology. 2013;144:528-35.

71. Sutcliffe P, Connock M, Gurung T, et al. Aspirin in primary prevention of cardiovascular disease and cancer: a systematic review of the balance of evidence from reviews of randomized trials. PLoS One. 2013;8:e81970.

72. Avivi D, Moshkowitz M, Detering E, et al. The role of low-dose aspirin in the prevention of colorectal cancer. Expert Opin Ther Targets. 2012;16:S51-62.

73. Pratt S, Thompson VJ, Elkin EP, et al. The impact of upper gastrointestinal symptoms on nonadherence to, and discontinuation of, low-dose acetylsalicylic acid in patients with cardiovascular risk. Am J Cardiovasc Drugs. 2010;10:281-8.

74. Eyre H, Kahn R, Robertson RM, et al. Preventing cancer, cardiovascular disease, and diabetes: a common agenda for the American Cancer Society, the American Diabetes Association, and the American Heart Association. Circulation. 2004;109: 3244-55. 